\title{
Applying Modeling and Simulation to Predict Human Injury due to a Blast Attack on a Shipboard Environment
}

\author{
Brigid Jacobs $^{1)}$, Lee Ann Young ${ }^{2)}$, Dr. Howard Champion ${ }^{3)}$, Mary Lawnick ${ }^{3)}$, Dr. Michael Galarneau ${ }^{4)}$, \\ Vern Wing ${ }^{4)}$, Dr. William Krebs ${ }^{5)}$ \\ 1) MCR, 2) Applied Research Associates, 3) SimQuest, 4) Naval Health Research Center, 5) Office of Na- \\ val Research
}

\begin{abstract}
Computer models simulating blast effects on ship personnel are needed, but thus far, development of models has focused on simulating blast effects on ship structure and equipment. Thus, capability gaps exist in predicting the type and severity of injuries from surface or underwater weapon impact, estimating medical response requirements, and determining outcomes of patients. The Human Injury \& Treatment (HIT) model addresses these gaps. Algorithms are utilized for scoring the type and severity of injuries predicted, using a variety of existing and developing injury models. Additional algorithms determine the post-injury level of incapacitation by evaluating how a physical impairment can impact performance of a task. A manning model simulates movement of personnel aboard the ship (Young, Allen, \& Minks, 2011). It functions iteratively with the Tactical Medical Logistics (TML+) code, a medical response model predicting resource utilization and patient outcomes (Mitchell, 2004). Impact: HIT will help the Navy and commercial maritime interests anticipate medical response requirements resulting from blast attacks to a ship, and understand the impact of personnel loss on the crew's ability to perform damage control.
\end{abstract}

\section{INTRODUCTION}

For several years, the US Navy has focused on the development of computer models simulating the effects of explosions on ships and their equipment. Minimal effort, however, has been applied to developing models that are dependable in simulating the effects of shipboard explosions on its personnel. The need for this capability is evident and casualty modeling is required for satisfying federally-mandated Testing \& Evaluation (T\&E) requirements. This capability is also critical in determining medical response requirements and ensuring sufficient manning and cross-training of crew members (Young, Allen, \& Minks, 2011).

The Human Injury \& Treatment (HIT) model predicts human injury, incapacitation, and medical response requirements needed, as a result of a blast event aboard a ship. Specifically, this tool tracks, measures, and determines the severity of human injury and treatment requirements, the ability for personnel to return to duty, and medical outcomes of the injured personnel. HIT provides probabilistic roll-up outcomes for a large quantity of likely hit points and associated injury scenarios. Post-attack, the HIT analysis takes into account availability of medical personnel, medical resources (supplies), operating condition (battle engagement or normal sailing), training, injury progression (degradation of injuries before treatment), response to medical interventions, and other applicable confounders potentially modifying treatment given (Young, Allen, \& Minks, 2010).

Although a variety of injury models already exist for various aspects of explosion bio-effects, models to predict injuries from smoke inhalation and inertial loading (ship shock) need to be developed. To characterize the disparate types of injuries caused by a blast attack (burns, eardrum rupture, fragment penetration, etc.), a common taxonomy was required. This taxonomy provides sufficient information about the type and severity of injury to drive medical response algorithms.
To understand performance limitations, an algorithm characterizes incapacitation among the injured personnel who return to duty with some degree of impairment. Although HIT leverages a pre-existing medical response model and pre-existing injury models, a variety of new algorithms were required to adapt that model to a ship-board environment. Lastly, algorithms were required to model moving patients/personnel around the damaged ship (Young et al., 2011).

Combined, the major components of HIT enable analysts to anticipate medical response requirements associated with blast attacks aboard a ship, and to understand the impact of personnel loss on the ability of the crew to perform damage control.

\section{PRACTICE INNOVATION}

HIT is built within the Endgame Framework (EF) architecture. EF is object-oriented and provides various features that combined, have not been available within existing or previous vulnerability/survivability modeling simulations. HIT's architecture allows the development and integration of independent analytical components to perform Modeling \& Simulation (M\&S) exercises that are tailored to the needs of the analyst. Further, the EF architecture enables time-stamped event queuing with parametric and stochastic processing. As shown in Figure 1, HIT's advanced visualization and rendering features enable its architecture to work with large scenarios and detailed models (Young et al., 2010).

As shown in Figure 2, HIT takes the results of ship vulnerability assessments as input. The Dynamic System Mechanics Advanced Simulation (DYSMAS2) (Schittke et al., 1989) and Advanced Ship Analysis Program (Pileggi, 2003) tools are used by the Navy to characterize the environment created aboard a ship as a result of underwater and open air explosions, respectively. The results of the assessments are 
then loaded into the HIT model. Examples of loading environment data include: the number, mass and speed of fragments that have hit personnel; static overpressure timehistories; dynamic pressure time histories; and quasi-static pressure time-histories (Young et al., 2010). These data inputs set the stage for HIT's assessment of personnel injury and incapacitation status.

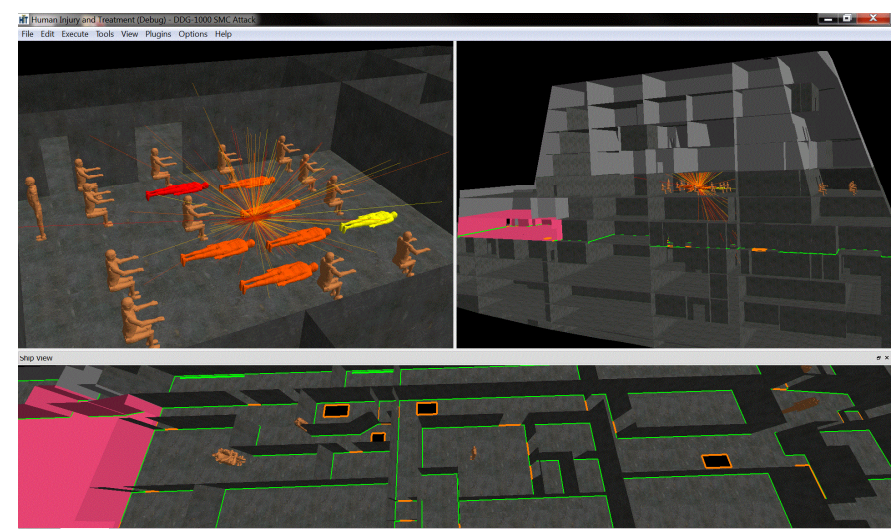

Figure 1 (Young et al., 2011). HIT Visualization of Damaged Ship. This figure illustrates the type of images produced during HIT's analysis of a damaged ship.

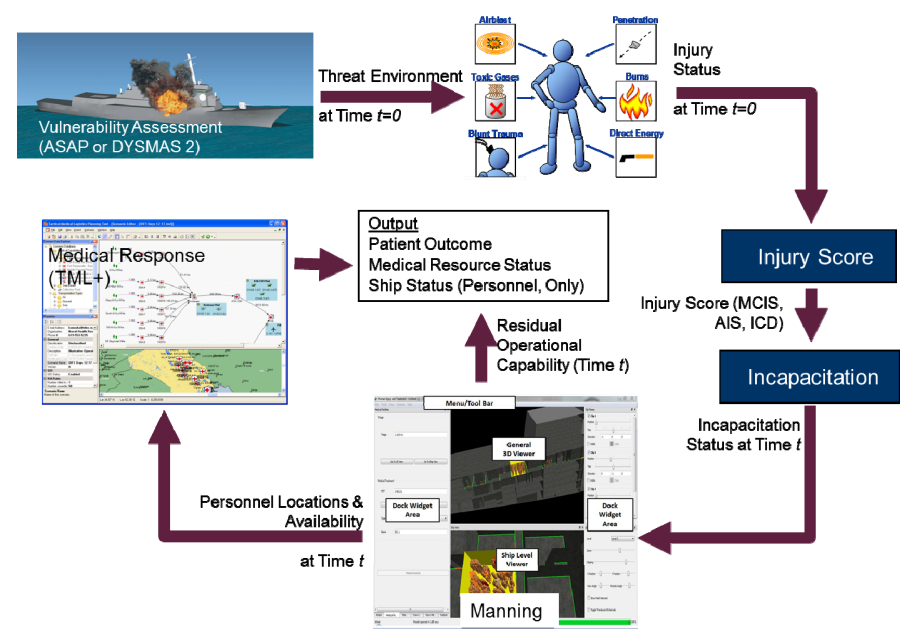

Figure 2 (Young et al., 2011). Major Components of HIT. This figure illustrates the major components of the HIT modeling and simulation tool.

Within HIT, the loading environment described above is used as input into the injury modules, so that the scoring of casualties (killed, injured, uninjured) and level of incapacitation (return to duty, medevac) can occur. Once the injury modules are called upon, the event-marching algorithm runs. Injuries are computed using a variety of injury models developed by the US Army, Navy, and Air Force to predict injuries from shock, fragments, debris, inertial ship motion, and fire (Young et al., 2010). The output from these modules is normalized into the Military Combat Injury Score (MCIS), which is a taxonomy for describing the type and severity of injuries interpreted by the medical response module (Champion \& Lawnick, 2012). The injury score is also used to assess incapacitation using the Military Functional Incapacity Score (MFIS), which characterizes the degree of impairment in terms of basic elements of function such as seeing, hearing, and moving (Champion \& Lawnick, 2012).

The level of incapacitation is computed by comparing the impairment of an individual with the functional requirements of that individual's job. The incapacitation data is used to present output relative to the remaining capabilities on a ship after the attack has occurred (Young et al., 2011). Thus, depending upon specific injuries and jobs, some wounded members aboard a damaged ship may be able to continue doing their originally assigned job. On the other hand, some who are injured may not be able to do their regular job, but can partake in other jobs as necessary.

The next major component of HIT is the manning module. This module oversees movement of personnel in order to accomplish medical tasks post-attack. Personnel movement is characterized as: movement from the point of injury to a location where self-aid or buddy-aid is administered, from the evacuation collection point, or from medical care duty (for return to duty patients) (Young et al., 2011).

Both the manning module and the medical response model, called Tactical Medical Logistics (TML+), are used iteratively. TML + simulates: patient progression through medical treatment facilities, medical resources available, and patient outcomes. TML+ provides the capability to determine the status of patients as a function of time. As these personnel are returned to duty they can be assigned roles in the ongoing damage control response within the manning module (Mitchell, 2004). TML+ has the capability to provide patient outcome for up to 72 hours. It also simulates injury status as a function of medical interventions (Young et al., 2011).

One of the major challenges of HIT is the development of a means to capture operational data, such as the operating condition and location of personnel at the time of the attack. Adequate information must be available to ensure a realistic HIT simulation without over-burdening the user. To meet this need, HIT includes a configuration utility. The ship configuration utility allows the user to position personnel in realistic locations before the attack, add important information to the ship (location of medical treatment facilities), and import manning data. The user can add and delete personnel and medical kits to explore the effects of these adjustments on the overall simulation. The ship configuration utility provides the capability of archiving ship and manning configurations for use in the future, as well as modifying existing configurations for fast creation of new scenarios (Young et al., 2011).

\section{FINDINGS}

The HIT modeling and simulation tool leverages existing models and methodologies wherever possible. However, to obtain the required level of fidelity, advancements are needed with respect to characterizing injuries and incapacitation, as well as, modeling medical response aboard a ship.

Existing taxonomies for quantifying injury types and severities have notable limitations. They are inadequate for characterizing important types of combat injury, like mandible fractures, open wounds with loss of more than $10 \%$ of muscle mass, and junctional vessel lacerations not amenable to tourniquet or compress solutions. Additionally, they cannot inte- 
grate multi-modal mechanisms of injury because their outputs are from a variety of models generated to classify injury as a result of experimental or simulated environments. To address these deficiencies, HIT has adopted and continued development of the MCIS system (Champion \& Lawnick, 2012).

The MCIS code is a five digit number that describes the severity and location of particular injuries. Each digit has a specific meaning and is defined in Table 1 . The first of the five digits identifies the severity of the injury. The scale for the first digit is from one to five (Table 1), with five being not survivable and one being a minor injury that should have no operational relevance. The second number identifies the injured region. For the MCIS code, the body has been divided into four body regions: (1) head and/or neck, (2) torso, (3) legs and (4) arms. The last three digits specify the specific injury. Note that an increase in the last three digits of the MCIS number is not proportional to the increase in severity of the injury (Champion \& Lawnick, 2012).

\begin{tabular}{|c|c|}
\hline Severity & Description \\
\hline $\begin{array}{c}1 \\
\text { Minor }\end{array}$ & $\begin{array}{l}\text { No immediate significant impairment in ability to shoot, move, or } \\
\text { communicate; likely to return to duty within } 72 \text { hours }\end{array}$ \\
\hline $\begin{array}{c}2 \\
\text { Moderate }\end{array}$ & $\begin{array}{l}\text { Possible impairment in immediate ability to shoot, move, or communicate; } \\
\text { likely able to contribute to sustaining mission, no in- } \\
\text { creased risk of death if treatment delayed due to tactical situation }\end{array}$ \\
\hline $\begin{array}{c}3 \\
\text { Serious }\end{array}$ & $\begin{array}{l}\text { No shock or airway compromise, ideally should be treated within } 6 \text { hours } \\
\text { of injury at a medical treatment facility (MTF) }\end{array}$ \\
\hline $\begin{array}{c}4 \\
\text { Severe }\end{array}$ & $\begin{array}{l}\text { Some will have increased risk of death or disability if not evacuated to an } \\
\text { MTF within } 6 \text { hours of injury }\end{array}$ \\
\hline $\begin{array}{c}5 \\
\text { Lethal }\end{array}$ & Injuries resulting in death immediately or within minutes \\
\hline
\end{tabular}

Table 1 (Champion \& Lawnick, 2012). MCIS Severity Scale. This scale represents the severity of personnel injuries.

Another shortfall with respect to existing taxonomies is that they are not correlated with measures of impairment. In contrast, the MCIS code is defined such that a severity of one constitutes no impairment, and levels of three through five constitute complete incapacitation. For injuries at severity level two there is some impairment. This impairment may or may not constitute incapacitation, as it is dependent upon the activities that the casualty needs to perform. To characterize incapacitation for level two injury severities, the MFIS defines the level of impairment according to the scale in Figure 3. To assess whether an individual is incapacitated, the functional requirements of the individual's task (firefighting), are compared to any injury impairments (Champion \& Lawnick, 2012). Thus, an individual with a sprained ankle will be incapacitated if his or her job requires mobility aboard a ship, but will not be incapacitated if his or her job requires sitting at a console.

The MCIS and MFIS scoring systems are still undergoing validation. MCIS is being validated using the Joint Theater Trauma Registry, the Tri-service Expeditionary Medical Encounter Database (EMED), and the Medical Examiners Trauma Registry (MTR). A panel of medical and operational personnel with shipboard experience is performing Face Validity of the MFIS (Champion \& Lawnick, 2012). Ongoing work includes development of an algorithm to quantify the overall severity of multiple, multi-modal injuries.
From a medical perspective, the primary function of MCIS is to drive the urgency and survival curves that are used in TML+ to determine treatment priorities and medical outcomes (Champion \& Lawnick, 2012). Figure 4 shows a survival curve generated with an existing taxonomy. Survival curves developed with MCIS should look similar, although they will be able to account for types of injuries not addressed with legacy trauma scoring systems.

\begin{tabular}{|c|c|c|c|}
\hline \multicolumn{2}{|c|}{ Vision } & \multicolumn{2}{|c|}{ Movement } \\
\hline 0 & No change in vision & 0 & No change in ability to move \\
\hline 1 & Impaired vision in one eye & 1 & Decreased ability to move \\
\hline 2 & Impaired vision in both eyes & 2 & Decreased ability to use one foot \\
\hline \multicolumn{2}{|c|}{ Hearing } & 3 & Decreased ability to use both feet \\
\hline 0 & No change in hearing & 4 & Loss of use of one foot \\
\hline 1 & Diminished hearing in one ear & 5 & Loss of use of both feet \\
\hline 2 & Diminished hearing in both ears & \multicolumn{2}{|c|}{ Strength/ Stamina } \\
\hline 3 & Dizziness or tinnitus & 0 & No change in strength/stamina \\
\hline \multicolumn{2}{|c|}{ Speech } & 1 & Impairment in ability to lift heavy objects; weakness \\
\hline \multirow{3}{*}{$\begin{array}{l}0 \\
1\end{array}$} & \multirow{3}{*}{$\begin{array}{l}\text { No change in speech } \\
\text { Impairment in ability to speak }\end{array}$} & & Impairment in ability to perform designated tasks for $\geq 1$ \\
\hline & & 2 & \\
\hline & & 3 & Unable to lift heavy objects \\
\hline \multicolumn{2}{|c|}{ Dexterity } & 4 & Unable to perform designated tasks for $<1$ hour \\
\hline 0 & No change in dexterity & \multicolumn{2}{|c|}{ Cognitive } \\
\hline 1 & Decreased ability to use one hand & 0 & No change in cognition \\
\hline 2 & Decreased ability to use both hands & 1 & Impairment in cognition \\
\hline 3 & Loss of use of one hand & & \\
\hline 4 & Loss of use of both hands & & \\
\hline
\end{tabular}

Figure 3 (Champion \& Lawnick, 2012). MFIS Impairments Scale. This scale characterizes incapacitation for level two injuries.

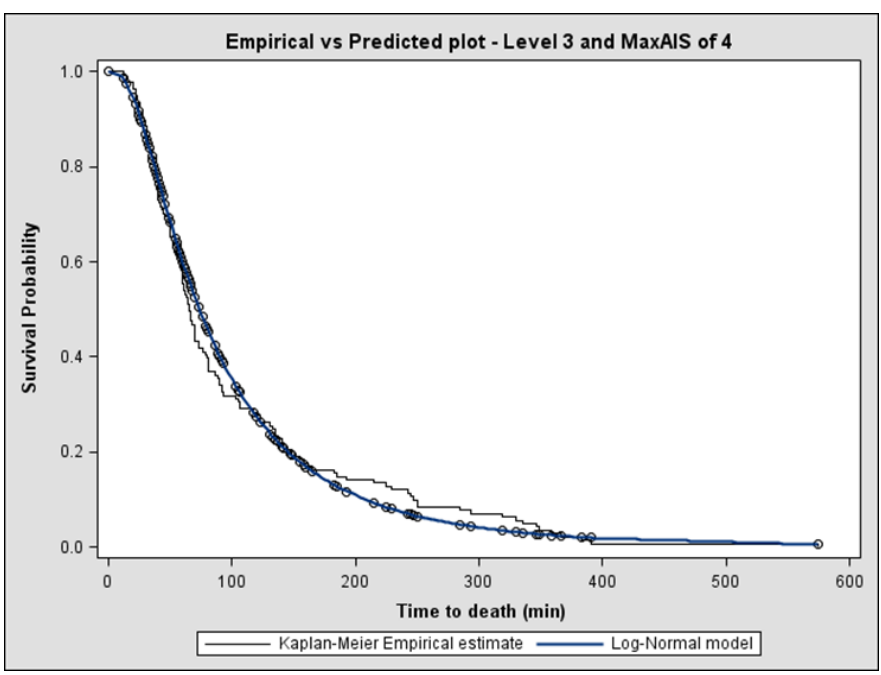

Figure 4. Sample Survival Curve. This figure provides an example of a survival curve, which helps determine treatment priorities and medical outcomes.

For any predictions within HIT to be valid, the underlying injury prediction models must yield reasonable and meaningful results. Presently, models exist for many of the effects that blasts have on the human body. However, there is currently no smoke inhalation model taking into account gases and particulates entrained in smoke. When combining burn and smoke inhalation, existing injury models lack quantifying short and long-term psychological effects. To address these issues, the HIT team has developed a construct for predicting injury severities as a function of exposure duration, clothing, fire temperature, and fire fuel. Existing burn and gas inhalation models will run in concert with a particulate inhalation model based upon test data available from cotton smoke inhalation. Where data gaps exist, a combination of heuristics and data from biological testing will be applied to ensure that fire and 
smoke dose-response relationships are characterized sufficiently. This will help to predict patient care requirements and treatment outcomes.

HIT produces both tabular and graphical reports related to injuries, incapacitation, and medical response. For stochastic runs, it reports out both a mean and a standard deviation of all output metrics, as well as, detailed results of individual iterations. For parametric runs, these results are post-processed using the Analysis of Variance techniques to reveal statistically significant differences among different trials.

With respect to injuries, HIT reports the type and severity of injuries based upon the MCIS severity scale. The types of injuries include: (1) penetration from fragments; (2) blast lung injury; (3) burns; (4) smoke inhalation; (5) blunt trauma from debris; (6) acceleration or blunt trauma from whole body displacement; and (7) temporary or permanent hearing loss. The severity of the injury is also described using MCIS, which ranges from one (no immediate impairment) to five (immediate death or death within minutes) (Champion \& Lawnick, 2012). As shown in Figure 5, an individual's injury or multiple injuries are assigned injury severity scores. For patients suffering from multiple injuries, an overall injury severity score is computed. This score takes into account the synergistic effects of the combined injuries. The injury results from HIT analyses can be used to generate Patient Condition Occurrence Frequency (PCOF) tables. These tables are then used by the medical logistics community to anticipate injuries and medical response requirements at the mission level.

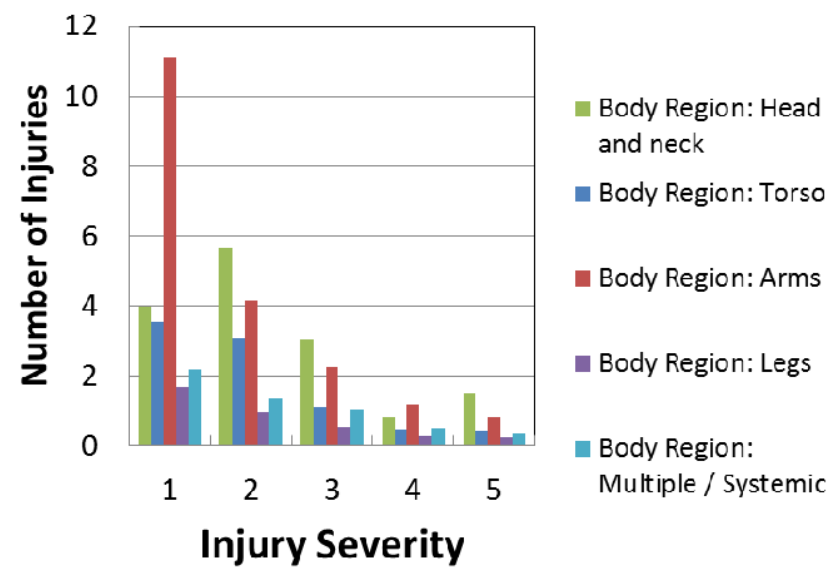

Figure 5. Injury Severity Scores. This figure serves as an example of potential injury severity scores that could be assigned to personnel injuries.

With respect to incapacitation, HIT reports the type and level of incapacitation for each crew member experiencing some level of impairment because of an injury. Incapacitation is defined relative to a job, and thus, incapacitation reports on individuals including not only their level of incapacitation on each scale, but the tasks for which they were incapacitated. HIT will also produce a report summarizing the billets/rates of lost personnel due to incapacitation (Figure 6).

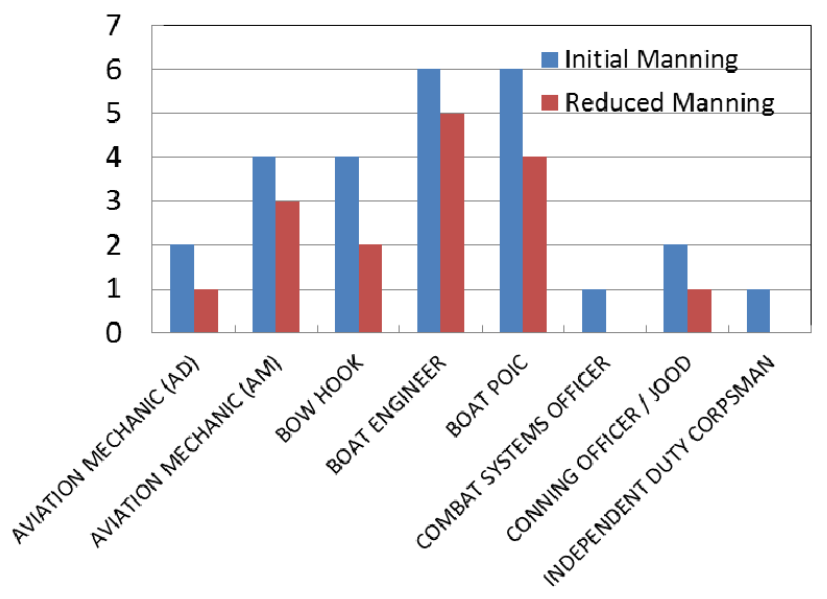

Figure 6. Billets/Rates of Lost Personnel. This figure provides an example summary of incapacitation effects on billets rates of lost personnel.

With respect to medical response, HIT produces output that TML+ users are accustomed to seeing (Mitchell, 2004). At the highest level, statistics are reported on the number of patients that are Killed in Action (KIA), Returned to Duty (RTD), or Evacuated from the ship for further treatment (Figure 7). However, more detailed HIT reports provide statistics on patients and include: disposition at each level of care, medical care throughput, consumable materials usage, equipment utilization, personnel utilization, and tasks performed.

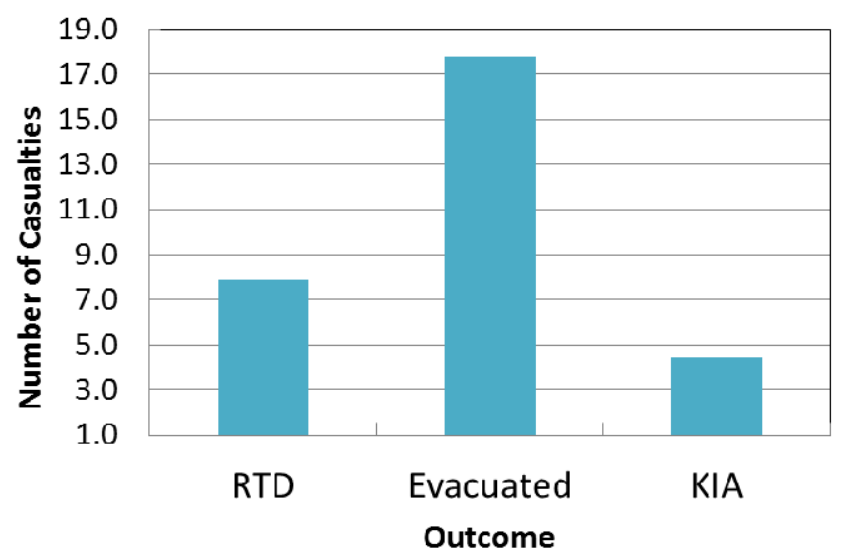

Figure 7. Distribution of Outcomes Across All Casualties. This figure provides an example of the number of casualties based on KIA, RTD, and Evacuation.

An important aspect of model development is validation. Four steps of Verification \& Validation $(V \& V)$ are used within the HIT tool. First, V\&V of leveraged submodels is inherited, and documentation is included within HIT. Second, the injury scoring model is V\&V against data contained in MTR and EMED. Third, incapacitation and medical response models are reviewed by Subject Matter Expert (SME) panels. Fourth, the overall HIT model will be used to emulate the USS Cole and USS Stark events. 


\section{DISCUSSION}

The HIT tool meets the needs of a number of Navy communities and is expected to have an impact on design of commercial maritime ships. Within the Navy, HIT will provide the Live Fire Test \& Evaluation (LFT\&E) community with a means to satisfy a Congressional requirement that addresses ship survivability in evaluation of ship design. Currently, crew member survivability is determined by SMEs who review realistic attack scenarios and then make educated guesses as to the number of casualties and the ultimate disposition (returned to duty, evacuated, deceased) of those casualties. HIT replaces those educated guesses with engineering models that can generate more detailed and supportable predictions. Additionally, HIT will assist in the design and planning of damage control trials, which will improve the overall realism of the crew's damage control response.

For the medical community, HIT's primary use will be to understand ships' medical response requirements. Currently, for medical planning purposes, this community also uses SMEs to predict the number and type of casualties that will occur for a given attack scenario. The SME predictions serve as inputs into the TML+ medical response simulation, which estimates medical response requirements in terms of equipment, supplies and personnel (Mitchell, 2004). With the introduction of HIT, the number, type, and severity of injuries will be predicted with the level of fidelity needed by TML + . Since HIT runs interactively with $\mathrm{TML}+$, the entire process from injury prediction to medical response requirements estimation will be completed without a man in the loop.

For manpower planning, HIT simulations will be capable of identifying post-attack losses in manning. With this information, manpower planners can determine the type of crosstraining or manning redundancies needed to execute damage control.

While the initial users of HIT will be within the US Navy, the applications described herein will also be of value to commercial ships as they become increasingly targeted by pirates and terrorists. Furthermore, HIT's architecture and data flow paradigm have been designed to facilitate adaptation of HIT, which meets changing and growing needs. For example, HIT can be expanded to predict injuries and simulate medical responses for chemical, biological, radiological, and nuclear attacks. HIT can also account for compounding effects of noncombat injuries and epidemics.

Some of the underlying Science \& Technology (S\&T) advancements in HIT have value beyond the Navy. The MCIS and MFIS scales were developed with medical and operational experts from across all three branches of the US Military. Ongoing MCIS validation uses combat injury data from these branches to ensure the taxonomy for characterizing injury severities is relevant for both land and ship-based conflicts (Champion \& Lawnick, 2012).

The MFIS was initially conceptualized as a metric for setting crew survivability design requirements for military vehicles. Impairments associated with blast injuries are evaluated relative to move, shoot, and communicate tasks. As implemented aboard a ship, the task list grew to include the myriad of activities associated with damage control (Champion \&
Lawnick, 2012). However, the fundamental principles of MFIS remain unchanged. It should be noted that the US Army has an alternative approach to characterizing incapacitation, leveraging the Operational Requirements-based Casualty Assessment (ORCA) model (Army Research Laboratory [ARL], 2008). Conceptually, the ORCA approach is similar, although it requires a more detailed characterization of tasks against which injury-induced impairments are assessed. It also provides a measure of incapacitation as a function of time. At this time, the database required to apply the ORCA incapacitation methodology lacks Navy jobs. However, should ORCA's database be extended to such jobs in the future, HIT's architecture has sufficient versatility to support either methodology.

The Died of Wounds curves are being generated using not only shipboard injury data, which is highly limited, but the near-census epidemiological and clinical data from the last decade of conflicts in the Middle East. The data involves Warfighters from all three branches of the US Military. It also ensures that TML + remains current in terms of functioning with both legacy and MCIS injury taxonomies, for both land and sea-based operations.

HIT's fire and smoke injury model will be applicable to both government and civilian organizations needing to predict injuries to personnel exposed to fire and smoke inside buildings, ships, or land-based vehicles. By taking into consideration gas and particulate inhalation effects of smoke, the HIT approach represents a substantial advancement in the state-of the-art smoke injury prediction. This model can also be used by state, local, and civilian medical response planners to anticipate the need for respirators critical to smoke inhalation survivability.

The beta release of the HIT software is scheduled for October 2012. As users begin to explore the HIT functionality, applications of the tool will likely increase, as will requests for additional functionality. Ultimately, HIT is expected to become an integral part of ship acquisition and medical planning processes for the Navy. Many of the S\&T efforts completed within the HIT program are expected to be used in both the Departments of Defense and Homeland Security.

\section{REFERENCES}

Army Research Laboratory. (2008). Operational requirements-based casualty assessment: ORCA user's manual. Aberdeen, MD.

Champion, H. R., \& Lawnick, M. (2012). Military combat injury scale (MCIS) and military functional incapacity scale (MFIS). Silver Spring, MD: SimQuest.

Mitchell, R. (2004). Modeling dynamic casualty mortality curves in the tactical medical logistics (TML+) planning tool. (Report No. 04-31). San Diego, CA: Naval Health Research Center.

Pileggi, M. (2003). Advanced survivability assessment program (ASAP) ecutive summary. Physical Mathematics and Computers, Inc.

Schittke, H.J., Mohr, W., Luetje, H., Pfrang, W.E., Freercks, J., \& Niessen, E. (1989). Proceedings from the 60th shock and vibration symposium IV: The program DYSMAS/ELC and its application on underwater shock loading of vessels. (pp.55-78).

Young, L., Allen, K., \& Minks, D. (2010). Human injury and treatment year 1 report. San Antonio, TX: Applied Research Associates.

Young, L., Allen, K., \& Minks, D. (2011). Human injury and treatment year 2 report. San Antonio, TX: Applied Research Associates. 